\title{
COMPOSITE S-BRANE SOLUTIONS ON PRODUCT OF RICCI-FLAT SPACES
}

\author{
V.D. Ivashchuk, V.N. Melnikov and A.B. Selivanov \\ Center for Gravitation and Fundamental Metrology, VNIIMS, 3-1 M. \\ Ulyanovoy Str., Moscow, 119313, Russia and \\ Institute of Gravitation and Cosmology, Peoples' Friendship University of \\ Russia, 6 Miklukho-Maklaya St., Moscow 117198, Russia \\ e-mails: ivas@rgs.phys.msu.su, melnikov@rgs.phys.msu.su, seliv@rgs.phys.msu.su
}

Key words: S-branes, cosmological solutions, extra dimensions, acceleration.

\begin{abstract}
A family of generalized $S$-brane solutions with orthogonal intersection rules and $n$ Ricci-flat factor spaces in the theory with several scalar fields and antisymmetric forms is considered. Two subclasses of solutions with power-law and exponential behaviour of scale factors are singled out. These subclasses contain sub-families of solutions with accelerated expansion of certain factor spaces. The solutions depend on charge densities of branes, their dimensions and intersections, dilatonic couplings and the number of dilatonic fields.
\end{abstract}




\section{Introduction}

The recent discovery of the cosmic acceleration [1, 2] was a starting point for a big number of publications on multidimensional cosmology giving some explanations of this phenomenon using certain multidimensional models [3], e.g. those of superstring or supergravity origin (see, for example [4] and references therein). These solutions deal with time-dependent scale factors of internal spaces (for reviews see [5, 6, 8, 9]) and contain as a special case the so-called S-brane solutions [10], i.e. space like analogues of $D$-branes [18, see for example [11, 12, 13, 14, 15, 16, 17] and references therein. For earlier $S$-brane solutions see also [19, 20, 21].

In our recent paper [4] we have obtained a family of cosmological solutions with $(n+1)$ Ricci-flat spaces in the theory with several scalar fields and multiple exponential potential when coupling vectors in exponents obey certain "orthogonality" relations. In [4 two subclasses of "inflationary-type" solutions with power-law and exponential behaviour of scale factors were found and solutions with accelerated expansion were singled out. In this paper we generalize "inflationary-type" solutions from [4] to $S$-brane configurations in models with antisymmetric forms and scalar fields. Two subclasses of these solutions with the power-law and exponential behaviour of scale factors in the synchronous time are singled out. These subclasses contain sub-families of solutions with accelerated expansion of certain factor spaces.

Here we deal with a model governed by the action

$$
S_{g}=\int d^{D} x \sqrt{|g|}\left\{R[g]-h_{\alpha \beta} g^{M N} \partial_{M} \varphi^{\alpha} \partial_{N} \varphi^{\beta}-\sum_{a \in \Delta} \frac{\theta_{a}}{n_{a} !} \exp \left[2 \lambda_{a}(\varphi)\right]\left(F^{a}\right)^{2}\right\}
$$

where $g=g_{M N}(x) d x^{M} \otimes d x^{N}$ is a metric, $\varphi=\left(\varphi^{\alpha}\right) \in \mathbf{R}^{l}$ is a vector of scalar fields, $\left(h_{\alpha \beta}\right)$ is a constant symmetric non-degenerate $l \times l$ matrix $(l \in \mathbf{N})$, $\theta_{a}= \pm 1, F^{a}=d A^{a}=\frac{1}{n_{a} !} F_{M_{1} \ldots M_{n_{a}}}^{a} d z^{M_{1}} \wedge \ldots \wedge d z^{M_{n_{a}}}$ is a $n_{a}$-form $\left(n_{a} \geq 1\right)$, $\lambda_{a}$ is a 1 -form on $\mathbf{R}^{l}: \lambda_{a}(\varphi)=\lambda_{\alpha a} \varphi^{\alpha}, a \in \triangle, \alpha=1, \ldots, l$. In (1.1) we denote $|g|=\left|\operatorname{det}\left(g_{M N}\right)\right|, \quad\left(F^{a}\right)_{g}^{2}=F_{M_{1} \ldots M_{n_{a}}}^{a} F_{N_{1} \ldots N_{n_{a}}}^{a} g^{M_{1} N_{1}} \ldots g^{M_{n_{a}} N_{n_{a}}}$, $a \in \triangle$. Here $\triangle$ is some finite set. For pseudo-Euclidean metric of signature $(-,+, \ldots,+)$ all $\theta_{a}=1$.

The paper is organized as following. In Section 2 we consider cosmologicaltype solutions with composite intersecting $S$-branes from [15, 9, 17] on product of Ricci-flat spaces obeying the "orthogonal" intersection rules. Section 3 is devoted to exceptional ("inflationary-type") $S$-brane solutions. 


\section{Cosmological-type solutions with compos- ite intersecting $p$-branes}

\subsection{Solutions with $n$ Ricci-flat spaces}

Let us consider a family of solutions to field equations corresponding to the action (1.1) and depending upon one variable $u$ [15] (see also [7, 8]).

These solutions are defined on the manifold

$$
M=\left(u_{-}, u_{+}\right) \times M_{1} \times M_{2} \times \ldots \times M_{n},
$$

where $\left(u_{-}, u_{+}\right)$is an interval belonging to $\mathbf{R}$, and have the following form

$$
\begin{array}{r}
g=\left(\prod_{s \in S}\left[f_{s}(u)\right]^{2 d\left(I_{s}\right) h_{s} /(D-2)}\right)\left\{\exp \left(2 c^{0} u+2 \bar{c}^{0}\right) w d u \otimes d u+\right. \\
\left.\sum_{i=1}^{n}\left(\prod_{s \in S}\left[f_{s}(u)\right]^{-2 h_{s} \delta_{i I_{s}}}\right) \exp \left(2 c^{i} u+2 \bar{c}^{i}\right) g^{i}\right\}, \\
\exp \left(\varphi^{\alpha}\right)=\left(\prod_{s \in S} f_{s}^{h_{s} \chi_{s} \lambda_{a_{s}}^{\alpha}}\right) \exp \left(c^{\alpha} u+\bar{c}^{\alpha}\right), \\
F^{a}=\sum_{s \in S} \delta_{a_{s}}^{a} \mathcal{F}^{s},
\end{array}
$$

$\alpha=1, \ldots, l ; a \in \triangle$.

In (2.2) $w= \pm 1, g^{i}=g_{m_{i} n_{i}}^{i}\left(y_{i}\right) d y_{i}^{m_{i}} \otimes d y_{i}^{n_{i}}$ is a Ricci-flat metric on $M_{i}$, $i=1, \ldots, n$,

$$
\delta_{i I}=\sum_{j \in I} \delta_{i j}
$$

is the indicator of $i$ belonging to $I: \delta_{i I}=1$ for $i \in I$ and $\delta_{i I}=0$ otherwise.

The $p$-brane set $S$ is by definition

$$
S=S_{e} \sqcup S_{m}, \quad S_{v}=\sqcup_{a \in \triangle}\{a\} \times\{v\} \times \Omega_{a, v},
$$

$v=e, m$ and $\Omega_{a, e}, \Omega_{a, m} \subset \Omega$, where $\Omega=\Omega(n)$ is the set of all non-empty subsets of $\{1, \ldots, n\}$. Here and in what follows $\sqcup$ means the union of nonintersecting sets. Any $p$-brane index $s \in S$ has the form

$$
s=\left(a_{s}, v_{s}, I_{s}\right)
$$

where $a_{s} \in \triangle$ is colour index, $v_{s}=e, m$ is electro-magnetic index and the set $I_{s} \in \Omega_{a_{s}, v_{s}}$ describes the location of $p$-brane worldvolume. 
The sets $S_{e}$ and $S_{m}$ define electric and magnetic $p$-branes, correspondingly. In (2.3)

$$
\chi_{s}=+1,-1
$$

for $s \in S_{e}, S_{m}$, respectively. In (2.4) forms

$$
\mathcal{F}^{s}=Q_{s} f_{s}^{-2} d u \wedge \tau\left(I_{s}\right),
$$

$s \in S_{e}$, correspond to electric $p$-branes and forms

$$
\mathcal{F}^{s}=Q_{s} \tau\left(\bar{I}_{s}\right),
$$

$s \in S_{m}$, correspond to magnetic $p$-branes; $Q_{s} \neq 0, s \in S$. Here and in what follows

$$
\bar{I} \equiv I_{0} \backslash I, \quad I_{0}=\{1, \ldots, n\} .
$$

All manifolds $M_{i}$ are assumed to be oriented and connected and the volume $d_{i}$-forms

$$
\tau_{i} \equiv \sqrt{\left|g^{i}\left(y_{i}\right)\right|} d y_{i}^{1} \wedge \ldots \wedge d y_{i}^{d_{i}},
$$

and parameters

$$
\varepsilon(i) \equiv \operatorname{sign}\left(\operatorname{det}\left(g_{m_{i} n_{i}}^{i}\right)\right)= \pm 1
$$

are well-defined for all $i=1, \ldots, n$. Here $d_{i}=\operatorname{dim} M_{i}, i=1, \ldots, n$; $D=1+\sum_{i=1}^{n} d_{i}$. For any set $I=\left\{i_{1}, \ldots, i_{k}\right\} \in \Omega, i_{1}<\ldots<i_{k}$, we denote

$$
\begin{array}{r}
\tau(I) \equiv \tau_{i_{1}} \wedge \ldots \wedge \tau_{i_{k}}, \\
d(I) \equiv \sum_{i \in I} d_{i}, \\
\varepsilon(I) \equiv \varepsilon\left(i_{1}\right) \ldots \varepsilon\left(i_{k}\right) .
\end{array}
$$

The parameters $h_{s}$ appearing in the solution satisfy the relations

$$
h_{s}=\left(B_{s s}\right)^{-1},
$$

where

$$
B_{s s^{\prime}} \equiv d\left(I_{s} \cap I_{s^{\prime}}\right)+\frac{d\left(I_{s}\right) d\left(I_{s^{\prime}}\right)}{2-D}+\chi_{s} \chi_{s^{\prime}} \lambda_{\alpha a_{s}} \lambda_{\beta a_{s^{\prime}}} h^{\alpha \beta},
$$

$s, s^{\prime} \in S$, with $\left(h^{\alpha \beta}\right)=\left(h_{\alpha \beta}\right)^{-1}$.

Here we assume that

$$
\text { (i) } \quad B_{s s} \neq 0 \text {, }
$$


for all $s \in S$, and

$$
\text { (ii) } \quad B_{s s^{\prime}}=0 \text {, }
$$

for $s \neq s^{\prime}$, i.e. canonical (orthogonal) intersection rules are satisfied.

The moduli functions read

$$
\begin{array}{r}
f_{s}(u)=R_{s} \sinh \left(\sqrt{C_{s}}\left(u-u_{s}\right)\right), C_{s}>0, h_{s} \varepsilon_{s}<0 ; \\
R_{s} \sin \left(\sqrt{\left|C_{s}\right|}\left(u-u_{s}\right)\right), C_{s}<0, h_{s} \varepsilon_{s}<0 ; \\
R_{s} \cosh \left(\sqrt{C_{s}}\left(u-u_{s}\right)\right), C_{s}>0, h_{s} \varepsilon_{s}>0 ; \\
\left|Q^{s}\right|\left|h_{s}\right|^{-1 / 2}\left(u-u_{s}\right), C_{s}=0, h_{s} \varepsilon_{s}<0,
\end{array}
$$

where $R_{s}=\left|Q_{s}\right|\left|h_{s} C_{s}\right|^{-1 / 2}, C_{s}, u_{s}$ are constants, $s \in S$.

Here

$$
\varepsilon_{s}=(-\varepsilon[g])^{\left(1-\chi_{s}\right) / 2} \varepsilon\left(I_{s}\right) \theta_{a_{s}},
$$

$s \in S, \varepsilon[g] \equiv \operatorname{sign}\left(\operatorname{det}\left(g_{M N}\right)\right)$. More explicitly (2.25) reads: $\varepsilon_{s}=\varepsilon\left(I_{s}\right) \theta_{a_{s}}$ for $v_{s}=e$ and $\varepsilon_{s}=-\varepsilon[g] \varepsilon\left(I_{s}\right) \theta_{a_{s}}$ for $v_{s}=m$.

Vectors $c=\left(c^{A}\right)=\left(c^{i}, c^{\alpha}\right)$ and $\bar{c}=\left(\bar{c}^{A}\right)$ obey the following constraints

$$
\begin{gathered}
\sum_{i \in I_{s}} d_{i} c^{i}-\chi_{s} \lambda_{a_{s} \alpha} c^{\alpha}=0, \quad \sum_{i \in I_{s}} d_{i} \bar{c}^{i}-\chi_{s} \lambda_{a_{s} \alpha} \bar{c}^{\alpha}=0, \quad s \in S, \\
c^{0}=\sum_{j=1}^{n} d_{j} c^{j}, \quad \bar{c}^{0}=\sum_{j=1}^{n} d_{j} \bar{c}^{j}, \\
\sum_{s \in S} C_{s} h_{s}+h_{\alpha \beta} c^{\alpha} c^{\beta}+\sum_{i=1}^{n} d_{i}\left(c^{i}\right)^{2}-\left(\sum_{i=1}^{n} d_{i} c^{i}\right)^{2}=0 .
\end{gathered}
$$

Here we identify notations for $g^{i}$ and $\hat{g}^{i}$, where $\hat{g}^{i}=p_{i}^{*} g^{i}$ is the pullback of the metric $g^{i}$ to the manifold $M$ by the canonical projection: $p_{i}: M \rightarrow$ $M_{i}, i=1, \ldots, n$. An analogous agreement will be also kept for volume forms etc.

Due to (2.9) and (2.10), the dimension of $p$-brane worldvolume $d\left(I_{s}\right)$ is defined by

$$
d\left(I_{s}\right)=n_{a_{s}}-1, \quad d\left(I_{s}\right)=D-n_{a_{s}}-1,
$$

for $s \in S_{e}, S_{m}$, respectively. For a $p$-brane we have $p=p_{s}=d\left(I_{s}\right)-1$.

Restrictions on $p$-brane configurations. The solutions presented above are valid if two restrictions on the sets of composite $p$-branes are 
satisfied [7]. These restrictions guarantee the block-diagonal form of the energy-momentum tensor and the existence of the sigma-model representation (without additional constraints) 22.

The first restriction reads

$$
\text { (R1) } \quad d(I \cap J) \leq d(I)-2
$$

for any $I, J \in \Omega_{a, v}, a \in \triangle, v=e, m$ (here $\left.d(I)=d(J)\right)$.

The second restriction is following one

$$
\text { (R2) } d(I \cap J) \neq 0,
$$

for $I \in \Omega_{a, e}$ and $J \in \Omega_{a, m}, a \in \triangle$.

\subsection{Minisuperspace-covariant notations}

Here we consider the minisuperspace covariant relations from [5, 22] for the sake of completeness. Let

$$
\left(\bar{G}_{A B}\right)=\left(\begin{array}{cc}
G_{i j} & 0 \\
0 & h_{\alpha \beta}
\end{array}\right), \quad\left(\bar{G}^{A B}\right)=\left(\begin{array}{cc}
G^{i j} & 0 \\
0 & h^{\alpha \beta}
\end{array}\right)
$$

be, correspondingly, a (truncated) target space metric and inverse to it, where (see [23])

$$
G_{i j}=d_{i} \delta_{i j}-d_{i} d_{j}, \quad G^{i j}=\frac{\delta^{i j}}{d_{i}}+\frac{1}{2-D},
$$

and

$$
U_{A}^{s} c^{A}=\sum_{i \in I_{s}} d_{i} c^{i}-\chi_{s} \lambda_{a_{s} \alpha} c^{\alpha}, \quad\left(U_{A}^{s}\right)=\left(d_{i} \delta_{i I_{s}},-\chi_{s} \lambda_{a_{s} \alpha}\right)
$$

are co-vectors, $s=\left(a_{s}, v_{s}, I_{s}\right) \in S$ and $\left(c^{A}\right)=\left(c^{i}, c^{\alpha}\right)$.

The scalar product from 22 reads

$$
\left(U, U^{\prime}\right)=\bar{G}^{A B} U_{A} U_{B}^{\prime},
$$

for $U=\left(U_{A}\right), U^{\prime}=\left(U_{A}^{\prime}\right) \in \mathbf{R}^{N}, N=n+l$.

The scalar products for vectors $U^{s}$ were calculated in $[22]$

$$
\left(U^{s}, U^{s^{\prime}}\right)=B_{s s^{\prime}}
$$


where $s=\left(a_{s}, v_{s}, I_{s}\right), s^{\prime}=\left(a_{s^{\prime}}, v_{s^{\prime}}, I_{s^{\prime}}\right)$ belong to $S$ and $B_{s s^{\prime}}$ are defined in (2.18). Due to relations (2.20) $U^{s}$-vectors are orthogonal, i.e.

$$
\left(U^{s}, U^{s^{\prime}}\right)=0
$$

for $s \neq s^{\prime}$.

The linear and quadratic constraints from (2.26) and (2.28), respectively, read in minisuperspace covariant form as follows:

$$
U_{A}^{s} c^{A}=0, \quad U_{A}^{s} \bar{c}^{A}=0,
$$

$s \in S$, and

$$
\sum_{s \in S} C_{s} h_{s}+\bar{G}_{A B} c^{A} c^{B}=0
$$

\section{Special solutions}

Now we consider a special case of classical solutions from the previous section when $C_{s}=u_{s}=c^{i}=c^{\alpha}=0$ and

$$
B_{s s} \varepsilon_{s}<0
$$

$s \in S$.

We get two families of solutions written in synchronous-type variable with:

A) power-law dependence of scale factors for $B \neq-1$,

B) exponential dependence of scale factors for $B=-1$, where

$$
B=\sum_{s \in S} h_{s} \frac{d\left(I_{s}\right)}{D-2} .
$$

Remind that $h_{s}=\left(B_{s s}\right)^{-1}$.

\subsection{Power-law solutions}

Let us consider the solution corresponding to the case $B \neq-1$. The solution reads

$$
\begin{array}{r}
g=w d \tau \otimes d \tau+\sum_{i=1}^{n} A_{i} \tau^{2 \nu_{i}} \hat{g}^{i}, \\
\varphi^{\alpha}=\frac{1}{B+1} \sum_{s \in S} \chi_{s} h_{s} \lambda_{a_{s}}^{\alpha} \ln \tau+\varphi_{0}^{\alpha},
\end{array}
$$


where $\tau>0$,

$$
\nu_{i}=-\frac{1}{B+1} \sum_{s \in S} h_{s}\left(\delta_{i I_{s}}-\frac{d\left(I_{s}\right)}{D-2}\right),
$$

$i=1, \ldots, n$ and

$$
\left|h_{s}\right|\left(\prod_{i \in \bar{I}_{s}} A_{i}^{d_{i}}\right) \exp \left(2 \chi_{s} \lambda_{a_{s} \alpha} \varphi_{0}^{\alpha}\right)=Q_{s}^{2}|B+1|^{2},
$$

$s \in S$; and $A_{i}>0$ are arbitrary constants.

The elementary forms read

$$
\mathcal{F}^{s}=\frac{\left|h_{s}\right| A^{1 / 2}}{Q_{s}(B+1)|B+1|} \tau^{-(B+2) /(B+1)} d \tau \wedge \tau\left(I_{s}\right),
$$

$s \in S_{e}$, (for electric case) and forms

$$
\mathcal{F}^{s}=Q_{s} \tau\left(\bar{I}_{s}\right),
$$

$s \in S_{m}$, (for magnetic case). Here and in what follows $w= \pm 1, Q_{s} \neq 0$, $s \in S$, and $A=\prod_{i=1}^{n} A_{i}^{d_{i}}$.

We see that these solutions depend on charged densities of branes, their dimensions and intersections, dilatonic couplings and the number of dilatonic fields.

In the special case of electric $S$-branes of maximal dimension $d\left(I_{s}\right)=$ $D-1$ the metric and scalar fields are coinciding (up to notations) with the solutions obtained in [4] when signature restrictions (3.1) are obeyed. Since solutions from [4] contain a subfamily of solutions with accelerated expansion of factor spaces, we are led to non-empty set of solutions with "acceleration" in the model under consideration 24].

\subsection{Solutions with exponential scale factors}

Here we consider the solution corresponding to the case $B=-1$. The solution reads

$$
\begin{array}{r}
g=w d \tau \otimes d \tau+\sum_{i=1}^{n} A_{i} \exp \left(2 M \mu_{i} \tau\right) g^{i}, \\
\varphi^{\alpha}=-M \tau \sum_{s \in S} h_{s} \chi_{s} \lambda_{a_{s}}^{\alpha}+\varphi_{0}^{\alpha},
\end{array}
$$


where

$$
Q_{s}^{2} \exp \left(-2 \chi_{s} \lambda_{a_{s} \alpha} \varphi_{0}^{\alpha}\right)=\left|h_{s}\right| M^{2} \prod_{i \in \bar{I}_{s}} A_{i}^{d_{i}}
$$

$s \in S$

$$
\mu_{i}=\sum_{s \in S} h_{s}\left(\delta_{i I_{s}}-\frac{d\left(I_{s}\right)}{D-2}\right),
$$

$M$ is parameter and $A_{i}>0$ are arbitrary constants, $i=1, \ldots, n$.

The elementary forms read

$$
\mathcal{F}^{s}=\frac{\left|h_{s}\right| A^{1 / 2}}{Q_{s}} M^{2} e^{M \tau} d \tau \wedge \tau\left(I_{s}\right),
$$

for $s \in S_{e}$, and $\mathcal{F}^{s}=Q_{s} \tau\left(\bar{I}_{s}\right)$ for $s \in S_{m}$.

In the cosmological case $w=-1$ we get an accelerated expansion of factor space $M_{i}$ if and only if $\mu_{i} M>0$ [24]. We see again that these solutions depend on charge densities of branes, their dimensions and intersections, dilatonic couplings and the number of dilatonic fields.

\section{Conclusions}

In this paper we considered generalized $S$-brane solutions with orthogonal intersection rules and $n$ Ricci-flat factor spaces in the theory with several scalar fields and antisymmetric forms. We singled out subclasses of solutions with power-law and exponential behaviour of scale factors depending in general on charge densities of branes, their dimensions and intersections, dilatonic couplings and the number of dilatonic fields. These subclasses contain sub-families of solutions with accelerated expansion of certain factor spaces [24, e.g. those considered in our earlier paper [4] (with signature restrictions (3.1) imposed).

We note that in our approach the intersection rules for composite $S$ branes have a minisuperspace covariant form, i.e. they are formulated in terms of scalar products of brane $U$-vectors and generally (see [15]) are classified by Cartan matricies of (semi-simple) Lie algebras. The intersection rules considered in this paper correspond to the Lie algebra $A_{1}+\ldots+A_{1}$.

\section{Acknowledgments}


This work was supported in part by the Russian Ministry of Science and Technology, Russian Foundation for Basic Research (RFFI-01-02-17312-a) and Project DFG (436 RUS 113/678/0-1(R)). V.N.M. is grateful to Prof. J.-M. Alimi for the hospitality during his stay at LUTH, Observatory ParisMeudon, France.

\section{References}

[1] A.G. Riess, et al AJ 116, 1009 (1998).

[2] S. Perlmutter, et al ApJ 517, 565 (1999).

[3] V.N.Melnikov, Exact Solutions in Multidimensional Gravity and Cosmology III. CBPF-MO-03/02, Rio de Janeiro, 2002, 297 pp.

[4] V.D. Ivashchuk, V.N. Melnikov and A.B. Selivanov, Cosmological solutions in multidimensional model with multiple exponential potential, JHEP 0309 (2003) 059; hep-th/0309027.

[5] V.D. Ivashchuk and V.N. Melnikov, Multidimensional classical and quantum cosmology with intersecting $p$-branes, hep-th/9708157; J. Math. Phys., 39, 2866-2889 (1998).

[6] V.D. Ivashchuk and V.N. Melnikov, Multidimensional cosmological and spherically symmetric solutions with intersecting p-branes. In: Lecture Notes in Physics, Vol. 537, "Mathematical and Quantum Aspects of Relativity and Cosmology. Proceedings of the Second Samos Meeting on Cosmology, Geometry and Relativity held at Pythagoreon, Samos, Greece, 1998, eds: S. Cotsakis, G.W. Gibbons., Berlin, Springer, 2000,pp. 214-148

[7] V.D. Ivashchuk and S.-W. Kim, Solutions with intersecting p-branes related to Toda chains, J. Math. Phys., 41 (1) 444-460 (2000); hep-th/9907019.

[8] V.D. Ivashchuk and V.N. Melnikov, Exact solutions in multidimensional gravity with antisymmetric forms, topical review, Class. Quantum Grav. 18, R82R157 (2001); hep-th/0110274

[9] V.D. Ivashchuk, On exact solutions in multidimensional gravity with antisymmetric forms, In: Proceedings of the 18th Course of the School on Cosmology and Gravitation: The Gravitational Constant. Generalized Gravitational Theories and Experiments (30 April-10 May 2003, Erice). Ed. by G.T. Gillies, V.N. Melnikov and V. de Sabbata, (Kluwer Acad.Publ.), to be published; gr-qc/0310114. 
[10] M. Gutperle and A. Strominger, Spacelike branes, JHEP 0204, 018 (2002); hep-th/0202210.

[11] C.M. Chen, D.M. Gal'tsov and M. Gutperle, S-brane solutions in supergravity theories; Phys. Rev. D 66, 024043 (2002); hep-th/0204071.

[12] M. Kruczenski, R.C. Myers and A.W. Peet, Supergravity S-branes, JHEP 0205, 039 (2002); hep-th/0204144.

[13] S. Roy, On supergravity solutions of space-like Dp-branes, hep-th/0205198.

[14] N.S. Degger and A. Kaya, Intersecting S-brane solutions of $D=11$ supergravity, hep-th/0206057

[15] V.D. Ivashchuk, Composite S-brane solutions related to Toda-type systems, Class. Quantum Grav. 20, 261-276 (2003); hep-th/0208101.

[16] N. Ohta, Intersection rules for S-branes, Phys. Lett. B 558, 213 (2003); hep-th/0301095

[17] V.D. Ivashchuk, On composite S-brane solutions with orthogonal intersection rules, hep-th/0309027.

[18] J. Polchinski, Dirichlet-Branes and Ramond-Ramond charges, Phys. Rev. Lett. 75, 4724 (1995); hep-th/9510017.

[19] K. Behrndt and S. Forste, String Kaluza-Klein cosmology, Nucl. Phys. B 430 441 (1994) 441; hep-th/9403179 (see also hep-th/9312167, hep-th/9704013).

[20] A. Lukas, B.A. Ovrut and D. Waldram, Cosmological solutions in type II string theory, Phys. Lett. B 393, 65 (1997); hep-th/9608195.

[21] H. Lü, S. Mukherji, C.N. Pope and K.-W. Xu, Cosmological solutions in string theories, Phys. Rev. D 55 , 7926-7935 (1997); hep-th/9610107.

[22] V.D. Ivashchuk and V.N. Melnikov, Sigma-model for the Generalized Composite p-branes, hep-th/9705036; Class. Quantum Grav. 14, 3001-3029 (1997); Corrigenda ibid. 15 (12), 3941 (1998).

[23] V.D. Ivashchuk, V.N. Melnikov and A.I. Zhuk, On Wheeler-DeWitt Equation in Multidimensional Cosmology, Nuovo Cimento B 104, 575 (1989).

[24] V.D. Ivashchuk, V.N. Melnikov and A.B. Selivanov, in preparation. 